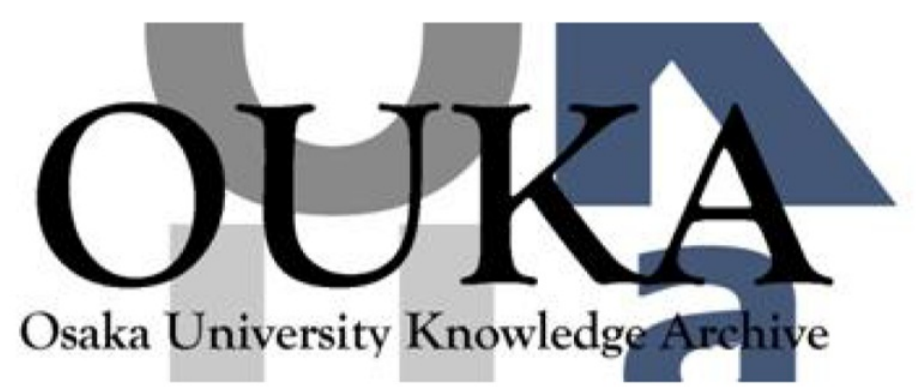

\begin{tabular}{|c|l|}
\hline Title & $\begin{array}{l}\text { On the behavior of ultraintense laser produced } \\
\text { hot electrons in self-excited fields }\end{array}$ \\
\hline Author(s) & Yabuuchi, T.; Adumi, K.; Habara, H. et al. \\
\hline Citation & Physics of Plasmas. 14(4) p. 040706 \\
\hline Issue Date & $2007-04$ \\
\hline oaire:version & VoR \\
\hline URL & https://hdl. handle. net/11094/2881 \\
\hline rights & \\
\hline Note & \\
\hline
\end{tabular}

Osaka University Knowledge Archive : OUKA

https://ir. Library. osaka-u. ac. jp/

Osaka University 


\title{
On the behavior of ultraintense laser produced hot electrons in self-excited fields
}

\author{
T. Yabuuchi, K. Adumi, H. Habara, R. Kodama, K. Kondo, T. Tanimoto, and K. A. Tanaka \\ Institute of Laser Engineering, Osaka University, 2-6, Yamada-oka, Suita, Osaka, 565-0871, Japan \\ and Graduate School of Engineering, Osaka University, 2-1, Yamada-oka, Suita, Osaka, 565-0871, Japan \\ Y. Sentoku \\ Department of Physics, University of Nevada, MS-220, Reno, Nevada 89557 \\ T. Matsuoka, ${ }^{\text {a) }}$ Z. L. Chen, ${ }^{\text {b) }}$ M. Tampo, A. L. Lei, and K. Mima \\ Institute of Laser Engineering, Osaka University, 2-6, Yamada-oka, Suita, Osaka, 565-0871, Japan
}

(Received 13 November 2006; accepted 13 March 2007; published online 27 April 2007)

\begin{abstract}
A large number of hot electrons exceeding the Alfvén current can be produced when an ultraintense laser pulse irradiates a solid target. Self-excited extreme electrostatic and magnetic fields at the target rear could influence the electron trajectory. In order to investigate the influence, we measure the hot electrons when a plasma was created on the target rear surface in advance and observe an increase of the electron number by a factor of 2 . This increase may be due to changes in the electrostatic potential formation process with the rear plasma. Using a one-dimensional particle-in-cell simulation, it is shown that the retardation in the electrostatic potential formation lengthens the gate time when electrons can escape from the target. The electron number escaping within the lengthened time window appears to be much smaller than the net produced number and is consistent with our estimation using the Alfvén limit. (C) 2007 American Institute of Physics.
\end{abstract}

[DOI: $10.1063 / 1.2722303$ ]

Relativistic electrons are generated when a target is irradiated with an ultraintense laser (UIL) pulse at an intensity of $10^{18} \mathrm{~W} / \mathrm{cm}^{2}$ or higher. These hot electrons have been widely used for the studies of electron beam acceleration, ${ }^{1-3}$ proton acceleration, ${ }^{4,5}$ and fast ignitor laser fusion. ${ }^{6,7}$ The characteristics of hot electrons have been studied such as conversion efficiency and slope temperature. The conversion efficiency as high as $40 \%$ has been observed in a target at intensities up to $10^{21} \mathrm{~W} / \mathrm{cm}^{2}{ }^{8,9}$ However, it is also known that the total energy of hot electrons observed in vacuum is only a few percent or less of the laser energy in experiments. ${ }^{10}$ The relation between the high production and the low observed efficiencies has not been fully understood. It is well known that very strong magnetic and electrostatic fields are excited in a vicinity of the target. These fields may affect the hot electrons departing from the target. Because of a return current in the target, the hot electron flow could exceed the Alfvén current, which is the maximum net current that can be sustained given the self-excited magnetic fields. ${ }^{11-13}$ When hot electrons depart from the target rear, a strong magnetic field should be excited in vacuum because there are no background electrons for the return current. The hot electron departure also excites an electrostatic field that can prevent the electron emission. ${ }^{14}$ It is known that the strength of the electrostatic field can reach few $\mathrm{MV} / \mu \mathrm{m}$ with a steep density profile at the target rear and can be reduced with a gradual density profile at the target rear. ${ }^{15}$ Both of the

\footnotetext{
${ }^{a)}$ Present address: Center for Ultrafast Optical Science, University of Michigan, Ann Arbor, Michigan 48109.

b) Present address: Department of Electrical and Computer Engineering, University of Alberta, Edmonton T6G 2V4, Canada.
}

strong fields may influence the hot electron trajectory and the escaping number when the hot electrons are emitted from the target-vacuum boundary.

In this Letter, we have studied the hot electron emission under the influence of self-excited fields at the target rear. In order to control the electrostatic field, a plasma is created on the target rear surface in advance. We show that the observed electron number increases twice with the rear plasma in our experiment when the target is irradiated with an UIL pulse. One-dimensional (1D) PIC simulation results show that the rear plasma retards the rise (gate closing) time of the electrostatic potential formation resulting in the increase of electrons escaping from the target. The electron number escaping within the gate time appears to be much smaller than the net produced number and is consistent with our estimation using the Alfvén limit.

The experiment is carried out using Gekko Module II (GMII) laser system at the Institute of Laser Engineering (ILE), Osaka University. ${ }^{16}$ The experimental setting is shown in Fig. 1. An UIL pulse $(\lambda=1053 \mathrm{~nm})$ is focused onto a foil target in $s$ polarization with $20^{\circ}$ incidence by an F/3.8 offaxis parabolic (OAP) mirror. The laser energy is changed from 2 to $10 \mathrm{~J}$ on the target and the maximum intensity is $3 \times 10^{18} \mathrm{~W} / \mathrm{cm}^{2}$ with a 700 fs pulse duration. The targets are aluminum plates with the thickness of 50-100 $\mu \mathrm{m}$. The long pulse laser $(\lambda=1053 \mathrm{~nm})$ with a $400 \mathrm{ps}$ duration is focused on the target rear surface with an energy of $1.5 \mathrm{~J}$, and creates a rear plasma in advance. The focus spot diameter of the long pulse is $150 \mu \mathrm{m}$ and is large enough compared to the UIL pulse focusing diameter of $20 \mu \mathrm{m}$. The density profile of the rear plasma is measured with an optical interferometric technique using a probe laser pulse $(\lambda=527 \mathrm{~nm})$ at $50 \mathrm{ps}$ before 


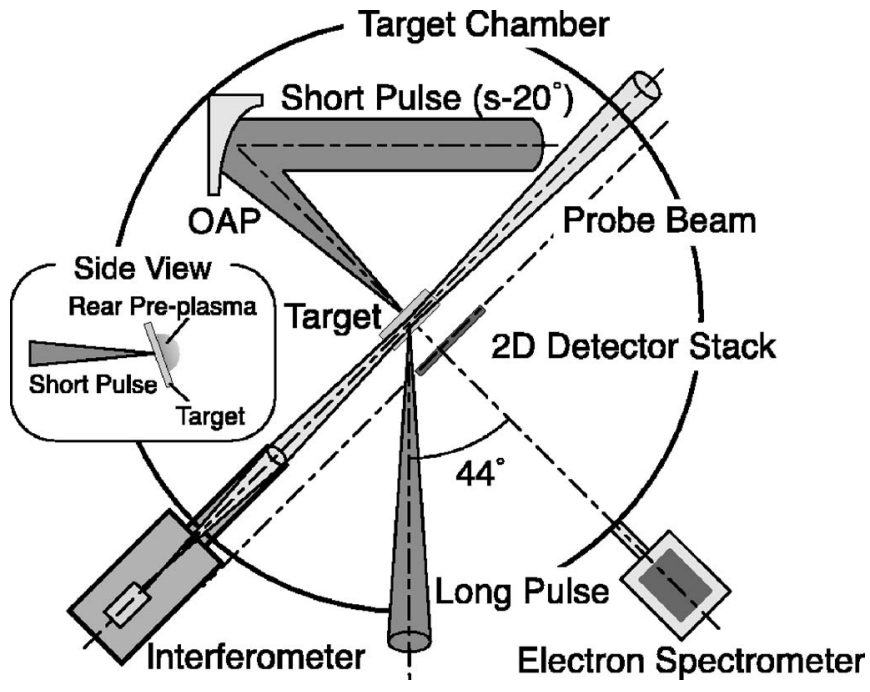

FIG. 1. Experimental setup (top view) The long pulse laser irradiates a target at $400 \mathrm{ps}$ before the UIL pulse. The target is tilted by $20^{\circ}$ for the $s$ polarized UIL irradiation. The interferogram is obtained at $50 \mathrm{ps}$ before the UIL pulse. Measured are the angular distributions of the hot electrons with IP and ions with CR-39 and RCF, and the energy spectrum of the hot electrons on the laser axis.

the UIL pulse. The detected density profile shows a good agreement with the results of a well calibrated 1D hydrodynamic simulation, ILESTA1D. ${ }^{17-19}$ The scale length of the rear plasma is $60 \mu \mathrm{m}$ at around the electron density of $10^{19} \mathrm{~cm}^{-3}$. We have confirmed that the shock wave generated by the long pulse does not reach the target front surface before the UIL pulse hits the target using the ILESTA1D simulation.

A two-dimensional (2D) angular distribution and an energy spectrum of hot electrons are simultaneously measured in order to estimate the total number of emitted hot electrons. An imaging plate (IP) is set at $40 \mathrm{~mm}$ away from the target as shown in Fig. 1 in order to measure the angular distribution. The IP is covered with several filters includes CR-39 and radiochromic film (RCF) to measure the proton angular distribution as well. The minimum energy of detectable electrons is $0.5 \mathrm{MeV}$ due to the filters. The energy spectrum of electrons is measured on the UIL axis with an electron spectrometer (ESM) using IP. ${ }^{20}$ For the ESM to catch the electrons, each layer of the stack detector has a 1-cm-diam hole on the laser axis. The total number of hot electrons emitted into the vacuum, $N_{\text {Tot }}$, is counted within the full width at half maximum (FWHM) of the angular distributions on the IP as follows. The distribution is fitted with two 2D Gaussian distributions in order to estimate the signal intensities within the FWHM, $I_{\text {FWHM }}$, and within the solid angle of the ESM at around the laser axis, $I_{\text {Axis }}$. It is known that the signal intensity on IP $(I)$ is proportional to the number of hot electrons $(N){ }^{21}$ Thus, $N_{\text {Tot }}$ has a relation of $N_{\text {Tot }} / I_{\text {FWHM }}=N_{\mathrm{ESM}} / I_{\text {Axis }}$. Here, $N_{\text {ESM }}$ is the absolute number of electrons obtained by the integration of the observed energy spectrum on the UIL axis. The sensitivity of IP used in the ESM is absolutely calibrated for a wide energy range of electrons. ${ }^{20}$

Typical experimental data are shown in Fig. 2 when the UIL pulse irradiated 50 - $\mu \mathrm{m}$-thick aluminum target at an in-
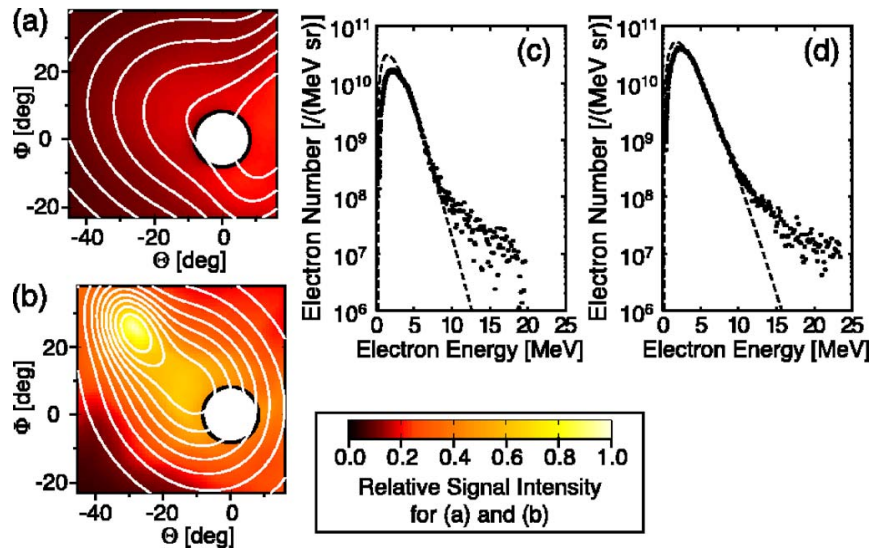

FIG. 2. (Color online) Angular distribution images of electrons (a) and (b) fitted with two 2D Gaussian distributions (contour lines) and energy spectra of hot electrons (c) and (d). (a) and (c) are obtained for a no rear plasma case. (b) and (d) are obtained for a rear plasma case. The energy spectra are fitted with the relativistic Maxwellian function (dashed curve).

tensity of $2 \times 10^{18} \mathrm{~W} / \mathrm{cm}^{2}$. The angular distributions of electrons are shown in Figs. 2(a) (without rear plasma) and 2(b) (with rear plasma). The white hole at $(\Theta, \Phi)=(0,0)$ corresponds to the laser axis in the distribution images. These images are normalized by the maximum signal intensity observed for the rear plasma case [Fig. 2(b)]. The fitting with two 2D Gaussian distributions is shown also in the figure as contour lines (every $10 \%$ of the maximum intensity in each figure). A bright spot of electron signal is seen in Fig. 2(b). The distribution could be influenced by the threedimensional plasma profile on the target rear surface. Figures 2(c) and 2(d) show the energy spectra of the electrons without and with rear plasma. The spectrum is fitted with a relativistic Maxwellian function, $N(\varepsilon)=\left(N_{0} / T\right)(\varepsilon / T)^{2} \exp (-\varepsilon / T)$, to determine the electron temperature, $T .{ }^{8}$ In the result, the temperature is $0.76 \pm 0.05 \mathrm{MeV}$ for the no rear plasma case and $0.85 \pm 0.04 \mathrm{MeV}$ for the rear plasma case. Since the shotto-shot variations of temperature are $\pm 0.1 \mathrm{MeV}$ in our experimental conditions, we see no difference in the temperature with and without rear plasma because of the same experimental conditions at the target front. The approximation with the relativistic Maxwellian function shows some difference from the experimental data at around the highenergy tail. However, the contribution to the field excitation may be negligible with these electrons because the electron number within the tail is at least two orders magnitude smaller than the one at around the peak of the spectrum. The energetic protons are observed at the target normal direction only when there is no rear plasma, consistent with Ref. 15.

The total number of electrons is estimated with the method described above. In the result, the $N_{\text {Tot }}$ is $5.7 \times 10^{10}$ and $1.2 \times 10^{11}$ for Figs. 2(a) and 2(b), respectively. The $N_{\text {Tot }}$ is plotted in Fig. 3(a) as a function of the electron temperature changing the UIL intensities from $2 \times 10^{17}$ to 3 $\times 10^{18} \mathrm{~W} / \mathrm{cm}^{2}$. The errors relevant to the electron number are $\sim 15 \%$ caused mainly by the interpolation of the angular distribution estimating $I_{\mathrm{FWHM}}$ and $I_{\text {Axis }}$. The Alfvén limit is also shown for an electron beam bunch with a $700 \mathrm{fs}$ time duration with an energy of $3 T$, which corresponds to the 

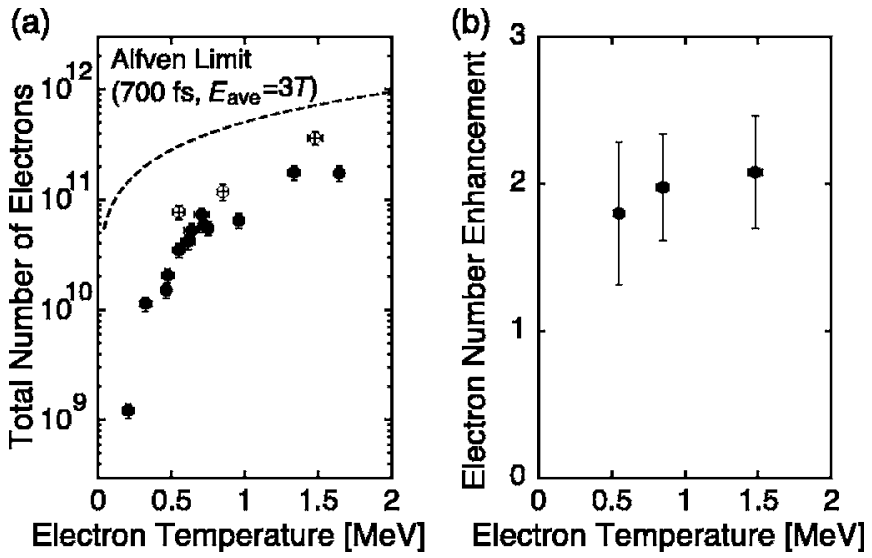

FIG. 3. (a) The number of hot electrons dependence on the electron temperature for no rear plasma cases $(\bullet)$ and for rear plasma cases $(\circ)$. The Alfvén limit is also shown (dashed curve). (b) Number increment of hot electrons versus the electron temperature due to the rear plasma.

average energy of the relativistic Maxwellian distributions with a temperature, $T$. The detected electron numbers are always smaller than the Alfvén limit. When the rear plasma exists, the number of detected electrons increases twice compared to the no rear plasma case at the similar electron temperature (UIL intensity) as shown in Fig. 3(b). Neither electrons nor ions are observed when the long pulse irradiates the target without the UIL pulse. Thus the observed electrons are purely due to the UIL pulse irradiation and the number enhancement is due to the presence of rear plasma.

In order to understand the influence of the rear plasma on the electrostatic potential formation, a 1D relativistic particle-in-cell simulation, PICLS1D, is performed. The PICLS1D code simulates the laser-plasma interactions using a numerical dispersion free Maxwell solver, and charge conservation scheme with Monte Carlo processes like Coulomb collisions. $^{22}$ Two different cases are considered in the simulations as shown in Fig. 4(a). In one case, the target has a sharp density boundary at the target rear (dashed line), and the other case is with a rear plasma of which scale length is $60 \mu \mathrm{m}$ at the target rear (solid line). The maximum density of the rear plasma is fixed to $0.7 n_{c}$, where $n_{c}$ is the critical density for a $1 \mu \mathrm{m}$ laser wavelength. In both cases, there is a preplasma in front of the target with the scale length $6 \mu \mathrm{m}$. The target density and thickness are also fixed to be $10 n_{c}$ and $50 \mu \mathrm{m}$. An UIL pulse enters the simulation box from the left boundary $(x=0)$ at time $t=0$ and irradiates the target at a maximum intensity $5 \times 10^{18} \mathrm{~W} / \mathrm{cm}^{2}$ with a Gaussian profile of $700 \mathrm{fs}$ duration in FWHM.

Because the surface plasma conditions at the front surface are the same for both cases, the characteristics of hot electrons are identical. A strong electrostatic field is excited at the target rear after the hot electrons start departing from the target. The profiles of the electrostatic field, $E_{x}$, are shown in Figs. 4(b) and 4(c) at the fixed timing when the field at the target rear reaches the maximum for the no rear plasma case. The maximum field strength is $1.5 \mathrm{MV} / \mu \mathrm{m}$ for the no rear plasma case [Fig. 4(b)]. The field reaches only $0.3 \mathrm{MV} / \mu \mathrm{m}$ for the rear plasma case [Fig. 4(c)].

Hot electrons may escape from the electrostatic potential
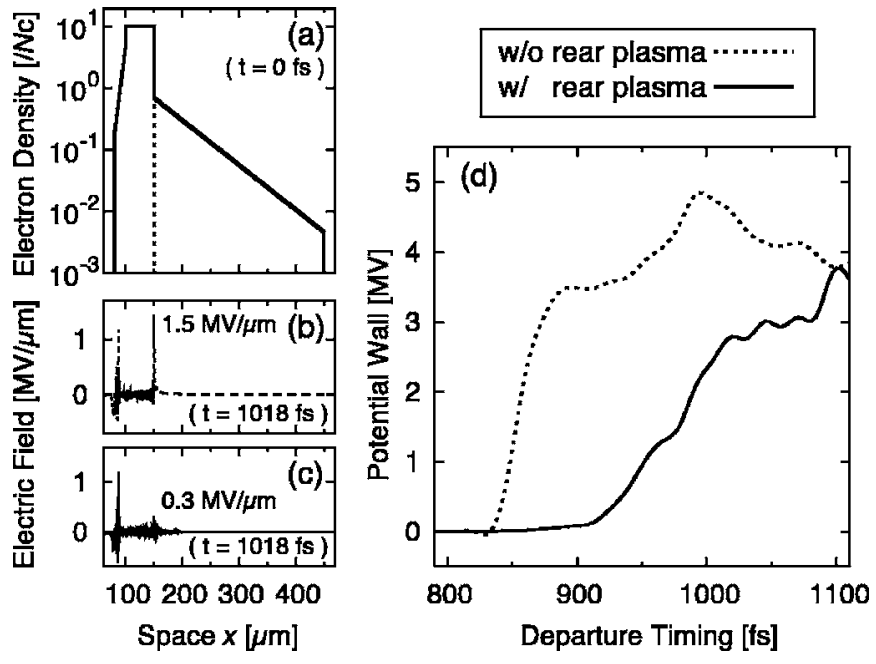

FIG. 4. (a) The initial density profile for PIC simulations. The rear boundary is sharp in a no rear plasma case (dashed curve) and the plasma exists at the target rear in another case (solid curve). (b), (c) The profile of the electrostatic field with rear plasma (b) and without rear plasma (c) when the field reaches a maximum for the no rear plasma case. (d) The potential wall for hot electrons is plotted as the function of the departure timing of the electrons.

until the potential exceeds the electron kinetic energy. The potential grows in time while the hot electrons depart from the target. This growth time may be considered as a gate closing time for escaping electrons. We estimate the potential evolution with time as following methods. The local potential is expressed as a spatial integration of the electrostatic field, $E_{x}\left(t_{i}, x\right)$, in a small interval from $x_{i}\left(t_{i}\right)$ to $x_{i+1}\left(t_{i+1}\right)$. Here, a hot electron is assumed to exist $x_{i}$ at $t_{i}$ and travel with the speed of light, $c$. Thus, $x_{i+1}=c\left(t_{i+1}-t_{i}\right)+x_{i}$. We summed the local potential by taking into account of both electron motion and field evolution in time space. A total potential, $\phi_{\text {Tot }}$, i.e. a potential wall, is expressed as Eq. (1) for an electron which departs the target rear boundary $(x$ $=150 \mu \mathrm{m})$ at a certain timing $t_{0}$,

$$
\phi_{\mathrm{Tot}}\left(t_{0}\right) \simeq \sum_{t_{i} \geq t_{0}} \int_{x_{i}}^{x_{i+1}} E_{x}\left(t_{i}, x\right) \mathrm{d} x .
$$

The potential wall evolves as shown in Fig. 4(d). The potential grows within 50 fs and reaches $\sim 4 \mathrm{MeV}$ and appears to be saturated for the no rear plasma (dashed curve). The potential growth is retarded for the rear plasma (solid curve) by 100-150 fs compared to the no rear plasma case.

We consider that the retardation is closely related to the charge neutralization due to a return current in the rear plasma. We estimate now the retardation time related to the time duration supplying the return current. The return current can be supplied until the number of hot electrons departing from the target becomes comparable to the electron number in the rear plasma. When the return current is assumed to flow in the opposite direction of the hot electron flow, the relation of electron number between the hot electrons and the return current can be simplified to the relation of the areal density of these electrons. Here, the areal density of hot electrons within the retardation time, $\Delta t$, is expressed as $n_{h} v_{h} \Delta t$, where $n_{h}\left(v_{h}\right)$ is the density (velocity) of hot electrons. The 
areal density of rear plasma is expressed as $n_{p} L$, where $n_{p}(L)$ is the peak density (scale length) of the rear plasma. Then the retardation time can be estimated as $\Delta t$ $=\left(n_{p} L\right) /\left(n_{h} v_{h}\right)$. Since $n_{h}$ and $v_{h}$ may be assumed to be $n_{c}$ and $c$, the retardation time becomes 140 fs for the plasma in Fig. 4(a) $\left(L=60 \mu \mathrm{m}\right.$ and $\left.n_{p}=0.7 n_{c}\right)$. This result is consistent with the retardation seen in Fig. 4(d). We performed simulations using different rear plasma scale lengths and confirmed the retardation time well agreed with the above argument.

The retardation of the potential evolution results in the lengthened gate time for electron escape. However, the hot electron flow produced by the UIL pulse could be larger than the Alfvén current. Thus the hot electrons escaping from the target within the lengthened gate time (140 fs) should be affected also by the Alfvén limit. The electron number corresponding to the Alfvén current is $8 \times 10^{10}$ for $2.5 \mathrm{MeV}$ electrons with $140 \mathrm{fs}$ pulse duration. This electron number is almost same as the observed number increase in the experiments $\left(10^{10-11}\right)$. Here, the $2.5 \mathrm{MeV}$ energy corresponds to the averaged value of the electrons in the measured spectra shown in Figs. 2(c) and 2(d). On the other hand, the number of hot electrons produced by the UIL pulse within $140 \mathrm{fs}$ is estimated to be $10^{13}$ and is much larger than the number detected in the experiments. Here, the electron number produced per time is approximated as $n_{c} c \pi r^{2}$, where $r$ is the laser spot radius, $10 \mu \mathrm{m}$. These results strongly infer that the number of escaping electrons are also determined by the Alfvén limit even when the potential gate is open.

In conclusion, we have studied the influence of the selfexcited fields on the limitation of hot electrons departing from the target. The detected number of hot electrons increases twice with the rear plasma under the irradiation of UIL pulse at a laser intensity of $2 \times 10^{18} \mathrm{~W} / \mathrm{cm}^{2}$. 1D PIC simulation shows that the formation of the electrostatic potential is retarded due to the presence of the rear plasma. The retardation is estimated also by equalizing the areal densities of hot electrons and the ones of the rear plasma. This model estimation matches very well with the simulated results when the rear plasma scale length is changed as a parameter. The hot electrons escape from the target until the potential exceeds the electron kinetic energy, so that the retardation of the potential growth lengthens the gate time resulting in an increase in the number of escaping electrons. The enhanced number of escaping electrons is consistent with the Alfvén current within the lengthened gate time.

The authors are very grateful to the laser operation group of GMII and the group of the target and material science of ILE.

This work was supported by MEXT, Grant-in Aid for Creative Scientific Research (15GS0214). Part of this research is supported by the CUP on plasma and nuclear fusion operated by NIFS and founded by JSPS. T.M. and A.L.L. acknowledge the support by JSPS.

${ }^{1}$ T. Tajima and J. M. Dawson, Phys. Rev. Lett. 43, 267 (1979).

${ }^{2}$ W. L. Kruer and K. Estabrook, Phys. Fluids 28, 430 (1985).

${ }^{3}$ F. Brunel, Phys. Rev. Lett. 59, 52 (1987).

${ }^{4}$ S. C. Wilks, A. B. Langdon, T. E. Cowan, M. Roth, M. Singh, S. Hatchett, M. H. Key, D. Pennington, A. MacKinnon, and R. A. Snavely, Phys. Plasmas 8, 542 (2001).

${ }^{5}$ J. Fuchs, P. Antici, E. D’humières et al., Nat. Phys. 2, 48 (2006).

${ }^{6}$ M. Tabak, J. Hammer, M. E. Glinsky, W. L. Kruer, S. C. Wilks, J. Woodworth, E. M. Campbell, M. D. Perry, and R. J. Mason, Phys. Plasmas 1, 1626 (1994).

${ }^{7}$ R. Kodama, H. Shiraga, K. Shigemori et al., Nature (London) 418, 933 (2002).

${ }^{8}$ K. Yasuike, M. H. Key, S. P. Hatchett, R. A. Snavely, and K. B. Wharton, Rev. Sci. Instrum. 72, 1236 (2001).

${ }^{9}$ R. Kodama, K. Mima, K. A. Tanaka et al., Phys. Plasmas 8, 2268 (2001).

${ }^{10}$ G. Malka, M. M. Aleonard, J. F. Chemin et al., Phys. Rev. E 66, 066402 (2002).

${ }^{11}$ H. Alfvén, Phys. Rev. 55, 425 (1939).

${ }^{12}$ L. Gremillet, G. Bonnaud, and F. Amiranoff, Phys. Plasmas 9, 941 (2002).

${ }^{13}$ Y. Sentoku, K. Mima, Z. M. Sheng, P. Kaw, K. Nishihara, and K. Nishikawa, Phys. Rev. E 65, 046408 (2002).

${ }^{14}$ J. Schreiber, F. Bell, F. Grüner et al., Phys. Rev. Lett. 97, 045005 (2006).

${ }^{15}$ A. J. Mackinnon, M. Borghesi, S. Hatchett, M. H. Key, P. K. Patel, H. Campbell, A. Schiavi, R. Snavely, S. C. Wilks, and O. Willi, Phys. Rev. Lett. 86, 1769 (2001).

${ }^{16}$ Y. Kitagawa, R. Kodama, K. Takahashi et al., Fusion Eng. Des. 44, 261 (1999).

${ }^{17}$ H. Takabe, M. Yamanaka, K. Mima et al., Phys. Fluids 31, 2884 (1988).

${ }^{18}$ K. A. Tanaka, H. Hashimoto, R. Kodama, K. Mima, Y. Sentoku, and K. Takahashi, Phys. Rev. E 60, 3283 (1999).

${ }^{19}$ K. Takahashi, R. Kodama, K. A. Tanaka, H. Hashimoto, Y. Kato, K. Mima, F. A. Weber, T. W. Barbee Jr., and L. B. D. Silva, Phys. Rev. Lett. 84, 2405 (2000).

${ }^{20}$ K. A. Tanaka, T. Yabuuchi, T. Sato, R. Kodama, Y. Kitagawa, T. Takahashi, T. Ikeda, Y. Honda, and S. Okuda, Rev. Sci. Instrum. 76, 013507 (2005).

${ }^{21}$ A. Taniyama, D. Shindo, and T. Oikawa, J. Electron Microsc. 46, 303 (1997).

${ }^{22}$ Y. Sentoku, K. Mima, Y. Kishimoto, and M. Honda, J. Phys. Soc. Jpn. 67, 4084 (1998). 